\title{
Experimental Observations on the Feasibility of VLC-Based V2X Communications under various Environmental Deterrents
}

\author{
Khagendra Joshi, Neelabhro Roy, Gurinder Singh, Vivek Ashok Bohara, and Anand Srivastava \\ Department of Electronics and Communications Engineering \\ IIIT-Delhi, New Delhi-110020, India \\ khagendra@iiitd.ac.in, neelabhro16171@iiitd.ac.in, gurinders@iiitd.ac.in, vivek.b@iiitd.ac.in, and anand@iiitd.ac.in
}

\begin{abstract}
Vehicle-to-Everything (V2X) using Visible Light Communication (VLC) channels can be seen as an economically viable option to replace the existing modes of vehicular communications in the near future. In this paper, we have analyzed the performance of VLC based V2X communication under various environmental deterrents viz. Light Fog, Dense Fog, Light Smoke and Dense Smoke using a proof-of-concept testbed. A series of experiments were conducted to investigate the effects of environmental deterrents over VLC based Lineof-Sight as well as non-Line-of Sight V2X transmission with respect to distance and angular variations. On-Off-keying (OOK) modulation has been selected as the modulation scheme, as defined in VLC standard (IEEE 802.15.7) for the transmission of information bits between a transmitter LED and a photodiode receiver. The experimental results show the feasibility of VLC-based V2X systems with reliable data transmission under different environmental deterrents with a fairly good signal-tonoise ratio (SNR), even under dense-fog and smoke conditions where the attenuation in average optical power at the receiver, is quite high.
\end{abstract}

Index Terms-Visible Light Communications (VLC), Vehicleto-Everything (V2X) Communications, Intelligent Transport Systems, Signal to Noise Ratio (SNR)

\section{INTRODUCTION}

Visible Light Communications (VLC) [1], [2] use the luminance of LEDs for optical wireless data communication. White light LEDs are favorable in many promising ways like long lifetime, high brightness, and lower power consumption. In recent years, VLCs utilizing white LEDs have been explored as a means of improving short-range wireless communications. Visible Light Communications are highly secure and offer a vast unlicensed free frequency spectrum usage. Moreover, they are proven to be harmless to the human body.

Based on a World Health Organization (WHO) report, road accidents cause an average of 1.2 million deaths worldwide annually, with-one fourth of all deaths caused by injury. It was also reported that about 50 million persons are injured in traffic accidents [3]. If any preventive measures are not taken timely, then road death is likely to become the thirdleading cause of death by 2020. The need of hour is to adopt intelligent transport system (ITS) to avoid and reduce vehicular accidents as well as improve passenger safety. To this end, the visible light communication (VLC) using vehicle headlamp and taillight can play a vital role in enhancing road safety and can be seen as a more reliable and efficient way of transmitting traffic related information even under adverse weather conditions on the road.

A hardware prototype presented in [4] has shown the feasibility for short-range broadcasting using a white LED lamp, paving the way for it to be considered, for providing indoor short range communications and illumination simultaneously. An optical wireless communication (OWC) system developed by I.Takai et al. in [4] showcases an LED transmitter and a camera receiver for automotive uses. Thus, the feasibility of VLC has been suggested to facilitate V2X vehicular communications (V2I and V2V) by exploiting the use of LED lights currently used for illumination in vehicles.

Various experimental scenarios pertaining to dedicated short range communication (DSRC) scenarios are documented in [5]. Specifically, [5] reported the performance of the DSRC based radio modules for V2V Communications. In [6], authors have analyzed V2V Communications under varying signalto-noise ratio (SNR), delay spread, vehicle speed, etc. and presented a detailed study of the DSRC physical layer. From these DSRC based papers, it can be deduced that RF based DSRCs involve higher cost for On board unit (OBU) and road side Unit (RSU) installation. The VLC-based V2V are more advantageous in offering lower costs, and complexity by utilizing the existing LED head-lamps in vehicles. Further, unlike VLC based V2X, RF based DSRC link availability is severely affected due to significant attenuation caused by buildings and other obstacles, which in turn reduces the communication-distance between vehicles. Further, RF based DSRC also experiences unwanted packet collision and end-toend delay, particularly during high traffic congestion which is rarely observed case for VLC based DSRC [7].

In [8], the authors suggest the use of a visible light communication (VLC) based model for V2V communications using off the shelf LEDs and photo diodes. In order to avoid road accidents, warning messages sent using the light from the brake lamps of a vehicle can facilitate precautionary measures (such as emergency hard brake). The adverse weather conditions such as dense fog, smog, rain, haze etc can severely affect VLC based V2V communications. In [9], the authors present the challenges, potential applications, and the experimental demonstration of Visible Light Communication (VLC) based 
Vehicle-to-Vehicle communications under the influence of fog condition.

Shen et al. in [10] have presented the experimental findings of the first ever real world driving test of a V2V making use of VLC channel prototype. It has been claimed that the angle and the distance between the transmitter and the receiver are the factors of primal importance in determining the received power and the related error performances.

In [11], the authors present a vehicle to vehicle VLC based $\mathrm{V} 2 \mathrm{~V}$ system comprising of LED-transmitter and an image sensor (camera) receiver. Optical communication image sensor (OCI) has been used in this paper, and achieves a throughput of $55 \mathrm{Mbps}$ for VLC signal transmission. The data rate was found to be faster than most RF based V2V and V2I communication systems.

In [12], the authors document the effects of fog and rain on the performance of vehicular visible light communications. A path loss model for $\mathrm{V} 2 \mathrm{~V}$ links as a function of distance for different environmental conditions has been proposed. The maximum achievable distance for such VLC based V2X scenario under various weather condition has been obtained theoretically without any experimental finding.

Motivated by the above mentioned, in this paper, we have analyzed the performance of VLC based V2X communication under various environmental deterrents viz. Light Fog, Dense Fog, Light Smoke and Dense Smoke using a proof-of-concept testbed.A series of experiments were conducted to investigate the effects of these environmental deterrents over VLC based line-of-sight as well as non-Line-of-sight V2X transmissions. The performance of the VLC communication link for line-ofsight and non-line-of-sight transmission has been evaluated in terms of the received SNR, with variation in distances and angles between the transmitter and receiver.

The organization of the paper is as follows. The VLC based $\mathrm{V} 2 \mathrm{~V}$ communication experiment has been explained in section 2. Section 3 discusses the design and development of system architecture for the testbed. The results have been discussed in section 4 and finally, useful conclusion and remarks have been made in section 5 .

\section{VLC BASED V2V COMMUNICATION EXPERIMENT}

The VLC based V2X communications can play a vital role in enhancing road safety and can be seen as being more reliable and efficient in transmitting traffic related information even under adverse weather conditions. We focus on investigating the effects of different environmental deterrents such as smoke and fog in the $\mathrm{V} 2 \mathrm{~V}$ transmission. The range and reliability of VLC based V2X communications are affected severely by these environmental deterrents. Fig. 1 shows the VLC-based V2V transmission model. This is analogous to the situation, when a driver encounters environmental deterrents on the road, the driver will usually switch on the vehicle's headlamp, fog lamps, or both. As a consequence, Car A with car headlamp as the VLC transmitter sends information to Car B as the receiver, with photodetector installed near its taillight. The present study primarily focuses on investigating the impacts of environmental deterrents on VLC based V2V along Line-of-sight as well as Non Line-of-sight transmission.

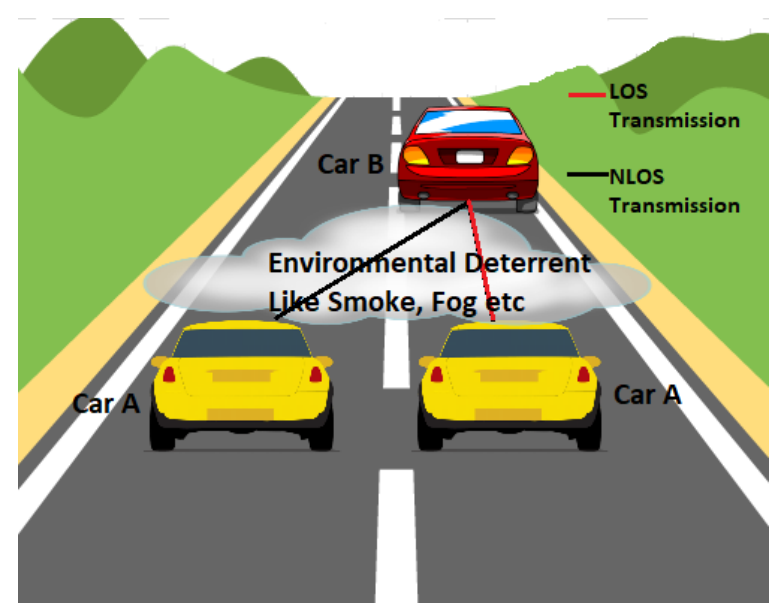

Fig. 1. VLC based V2V transmission model. Here, Car A with car headlamp as the transmitter, is trying to send information to Car B as the receiver, with the photodetector installed near its taillight.

\section{Design And DeVelopment of System ARCHITECTURE}

\section{A. System Architecture}

The basic system architecture consists of the VLC transmitter and the receiver section as shown in Fig. 2. The transmitter section includes the Host PC with LabVIEW API running on it, universal software radio peripheral (USRP N210) with low frequency transmitter (LFTX) daughterboard (USRP TX) and an LED driver circuit to drive the commercial off the shelf (COTS) high power (1W) white LED. The LFTX ensures a clean operation of the USRP from DC to $30 \mathrm{MHz}$ by employing high-speed operational amplifiers. A Bias tee is used to add a DC offset to the output signal of the USRP TX to a desired threshold voltage for the switching MOSFET used in the LED driver circuit. The LED driver circuit consists of high switching MOSFET. The N-type Enhancement Power MOSFET (IRFZ44N) has been used for switching the 1 watt LEDs with high current.

The receiver section includes a PDA36A2, which is a switchable-gain photo detector especially designed for distinguishing light signals with wavelengths ranging from $350 \mathrm{~nm}$ to $1100 \mathrm{~nm}$. The gain can be adjusted over a range of $70 \mathrm{~dB}$ which is possible due to the presence of a high gain and low offset trans-impedance amplifier. The output of the PDA36A2 is fed to the USRPN210 with the low frequency receiver (LFRX) daughterboard. The LFRX provides a reception coverage from DC to $30 \mathrm{MHz}$ by utilizing its high-speed operational amplifier. The LFRX is the best possible solution for the users who aim to directly receive the signals in the high frequency band utilizing an external Low Noise Amplifier (LNA), or use an external front-end for getting a down converted signal. The current study shows implementation which supports On-off Keying (OOK) modulation and demodulation. 


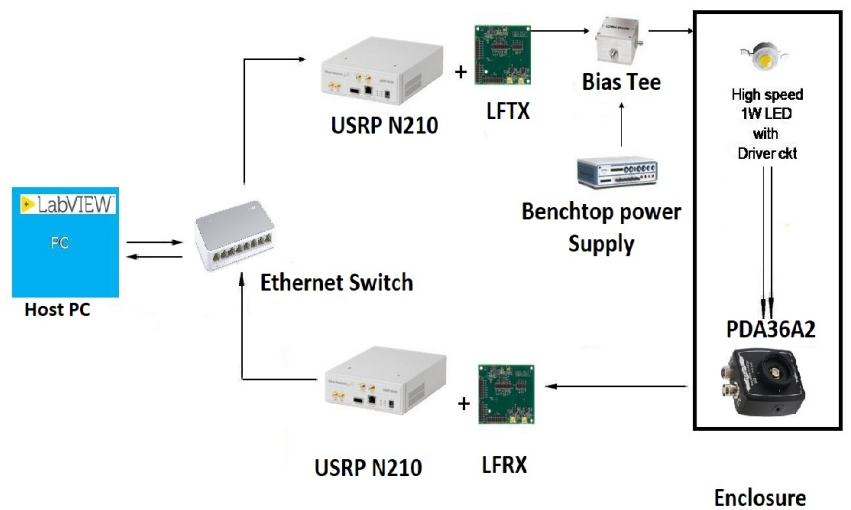

Fig. 2. System Architecture

\section{B. Proof-of-concept Experimental set up}

A proof-of-concept experimental testbed has been shown in Fig. 3. The idea behind the testbed is to emulate a real-world transportation scenario, wherein we can study and analyze the effects of V2X transmission on roads under the impact of various environmental deterrents like smoke, fog etc. Our testbed is an enclosure made up of acrylic material with dimension $122 \times 112 \times 25 \mathrm{~mm}$. For simulating a smoky environment, we have used BD-1500, which is a smoke generator machine. A foggy environment was developed using a chemical combination of dry ice and hot water. The performance of the VLC communication link for Line of sight and non-line of-sight transmission has been evaluated in terms of received SNR with variation in distances and angles between the transmitter and receiver. Few important experimental parameters have been shown in Table I.

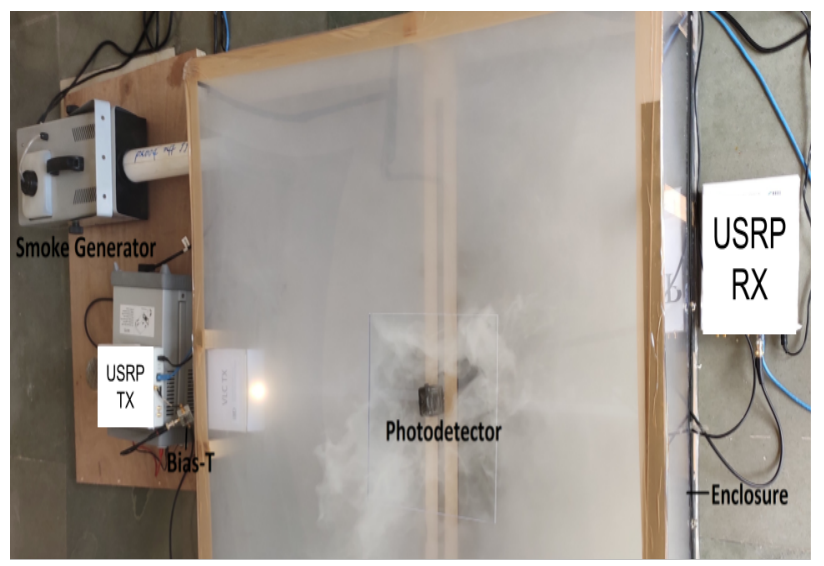

Fig. 3. Experimental Set up under Smoke condition.

\section{RESULTS AND DISCUSSION}

We conducted multiple tests on our VLC based V2X testbed. Primarily, we studied the SNR for our data transmission, in the absence of strong ambient light, so as to obtain the best results. We performed an extensive distance
TABLE I

EXPERIMENTAL PARAMETERS

\begin{tabular}{|l|l|l|}
\hline S.No. & System Parameters & Values \\
\hline 1 & Transmission bit rate & $\begin{array}{l}250 \\
\text { Kbps }\end{array}$ \\
\hline 2 & $\begin{array}{l}\text { Communication Link } \\
\text { Distance }\end{array}$ & $1 \mathrm{~m}$ \\
\hline 3 & Modulation Scheme & OOK \\
\hline 4 & $\begin{array}{l}\text { Wavelength of White } \\
\text { LED }\end{array}$ & $625 \mathrm{~nm}$ \\
\hline 5 & $\begin{array}{l}\text { Power dissipation of } \\
\text { White LED }\end{array}$ & $1 \mathrm{~W}$ \\
\hline 6 & Detection area of PD & $13 \mathrm{~mm}^{2}$ \\
\hline 7 & Responsivity of PD & $0.3 \mathrm{~A} / \mathrm{W}$ \\
\hline
\end{tabular}

and angular analysis, under the influence of two environmental deterrents, namely, smoke and fog. We varied their densities inside the testbed in order to have a more detailed analysis. The observed typical values of attenuation of different environmental deterrent through measurement and empirical calculations based on [7], [9] can be summarized in Table II. It can be inferred from the table that dense smoke attenuates the received optical power, more severely at the receiver as compared to the other environmental deterrents due to its highest attenuation coefficient.

TABLE II

TYPICAL VALUES OF ATTENUATION (DB/KM) WITH VISIBILITY PARAMETER, V (KM)

\begin{tabular}{|l|l|l|l|}
\hline S.No. & $\begin{array}{l}\text { Environmental } \\
\text { Deterrent }\end{array}$ & $\begin{array}{l}\text { Visibility } \\
\text { Parameter, V } \\
(\mathbf{K m})\end{array}$ & $\begin{array}{l}\text { Attenuation } \\
\mathbf{( d B / k m )}\end{array}$ \\
\hline 1 & $\begin{array}{l}\text { No Environmen- } \\
\text { tal Deterrent }\end{array}$ & 21.2 & 0.69 \\
\hline 2 & Light Fog & 1 & 14.9 \\
\hline 3 & Dense Fog & 0.500 & 34.7 \\
\hline 4 & Light Smoke & 0.300 & 57.8 \\
\hline 5 & Dense Smoke & 0.150 & 115.6 \\
\hline
\end{tabular}

Fig. 4 represents the case in which the impact of angular variation in Non-Line of Sight transmission using VLC has been observed. The distance between transmitter and receiver is kept to be $40 \mathrm{~cm}$. Irrespective of any environmental deterrent conditions, the SNR remains almost constant upto $45^{\circ}$. It can also be observed that as expected, dense smoke affects the NLOS transmission more severely as compared to the other environmental deterrents which is mainly due to high attenuation in the received optical power at the receiver.

Fig. 5 represents the case in which the impact of the angular variation in Non-Line of Sight transmission using VLC was observed when distance between the transmitter and the receiver is kept to be $60 \mathrm{~cm}$. Irrespective of any environmental deterrent conditions, the SNR remains almost constant upto $30^{\circ}$. As compared to Fig. 4, SNR degrades more with angular variation in Non-Line of sight transmission.

Fig. 6 shows a variation of SNR across various distances for a Line of Sight transmission. As expected, the SNR degrades as distance between transmitter and receiver increases. It 


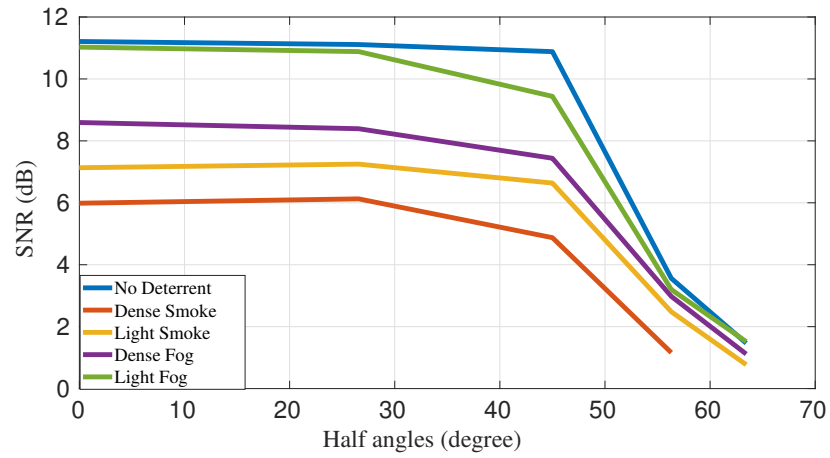

Fig. 4. Received SNR variation with half angles for NLOS transmission when distance between transmitter and receiver is $40 \mathrm{~cm}$ under no deterrent $(\mathrm{V}=21 \mathrm{Km})$, Light Fog $(\mathrm{V}=1 \mathrm{Km})$, Dense Fog $(\mathrm{V}=0.500 \mathrm{Km})$, Light Smoke $(\mathrm{V}=0.300 \mathrm{~km})$, and Dense Smoke $(\mathrm{V}=0.150 \mathrm{Km})$ conditions.

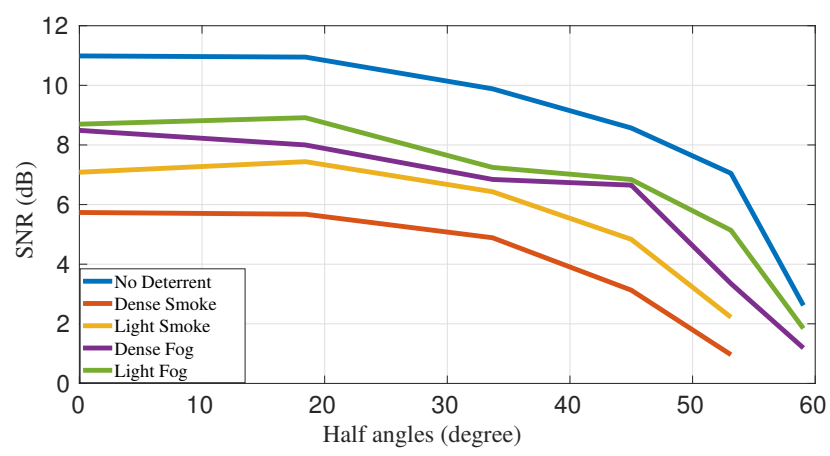

Fig. 5. Received SNR variation with half angles for NLOS transmission when distance between transmitter and receiver is $60 \mathrm{~cm}$.

can be seen that irrespective of any distance between the transmitter and the receiver, our test bed can achieve a stable SNR of around $11 \mathrm{~dB}$ without any environmental deterrent. The impact of different environmental deterrents on LOS transmission is more severe for dense smoke conditions which is again due to high attenuation in optical power received at the receiver.

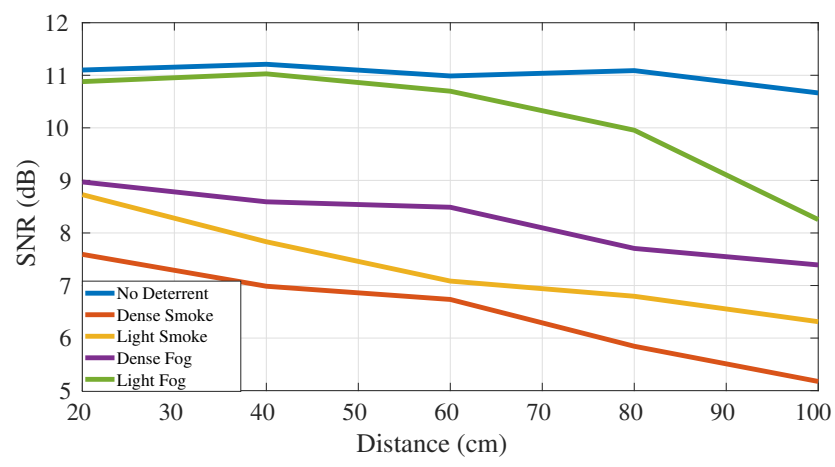

Fig. 6. Received SNR variation with distance between transmitter and receiver for LOS transmission

\section{Conclusion}

The performance of VLC-based V2X communications under the impact of various environmental deterrents such as dense smoke, light smoke, dense fog, and light fog have been analyzed using a proof-of-concept testbed. The VLC based line-of-sight and non-line-of-sight V2X communication was achieved using NI USRPs with customized low frequency daughter boards. It was found that in case of Non line-ofsight as well as light-of sight communication, the VLC link is severely affected by strong deterrent such as dense smoke. However, the impact of dense fog, light fog and light smoke on VLC communication link was less compared to dense smoke while maintaining fairly good SNR performance. From the experimental results, it can be inferred that the proposed VLCbased V2X framework presents an extremely viable potential candidate for future V2X communication systems with an increased understanding of the adverse effects of non-aligned vehicular communication.

\section{REFERENCES}

[1] T. Komine and M. Nakagawa, "Fundamental analysis for visible-light communication system using led lights," IEEE Transactions on Consumer Electronics, vol. 50, no. 1, pp. 100-107, Feb 2004.

[2] L. Zeng, D. C. O'Brien, H. L. Minh, G. E. Faulkner, K. Lee, D. Jung, Y. Oh, and E. T. Won, "High data rate multiple input multiple output (mimo) optical wireless communications using white led lighting," IEEE Journal on Selected Areas in Communications, vol. 27, no. 9, pp. 16541662, December 2009.

[3] H. D. M. Peden, Margie and E. Jarawan, "World report on road traffic injury prevention," in World Health Organization., June 2018, pp. 1-66.

[4] I. Takai, S. Ito, K. Yasutomi, K. Kagawa, M. Andoh, and S. Kawahito, "Led and cmos image sensor based optical wireless communication system for automotive applications," IEEE Photonics Journal, vol. 5, no. 5, pp. 6801418-6801418, Oct 2013.

[5] V. Kukshya and H. Krishnan, "Experimental measurements and modeling for vehicle-to-vehicle dedicated short range communication (dsrc) wireless channels," in IEEE Vehicular Technology Conference, Sep. 2006, pp. $1-5$.

[6] R. Sabouni and R. M. Hafez, "Performance of dsrc for v2v communications in urban and highway environments," in 2012 25th IEEE Canadian Conference on Electrical and Computer Engineering (CCECE), April 2012, pp. 1-5.

[7] G. Singh, A. Srivastava, and V. Bohara, "On feasibility of vlc based car-to- car communication under solar irradiance and fog conditions," Proceedings of the 1st International Workshop on Communication and Computing in Connected Vehicles and Platooning, pp. 1-7, Feb 2018.

[8] K. Siddiqi, A. D. Raza, and S. S. Muhammad, "Visible light communication for $\mathrm{v} 2 \mathrm{v}$ intelligent transport system," in 2016 International Conference on Broadband Communications for Next Generation Networks and Multimedia Applications (CoBCom), Sep. 2016, pp. 1-4.

[9] Y. H. Kim, W. A. Cahyadi, and Y. H. Chung, "Experimental demonstration of vlc-based vehicle-to-vehicle communications under fog conditions," IEEE Photonics Journal, vol. 7, no. 6, pp. 1-9, Dec 2015.

[10] W. Shen and H. Tsai, "Testing vehicle-to-vehicle visible light communications in real-world driving scenarios," in 2017 IEEE Vehicular Networking Conference (VNC), Nov 2017, pp. 187-194.

[11] Y. Goto, I. Takai, T. Yamazato, H. Okada, T. Fujii, S. Kawahito, S. Arai, T. Yendo, and K. Kamakura, "A new automotive vlc system using optical communication image sensor," IEEE Photonics Journal, vol. 8, no. 3, pp. 1-17, June 2016.

[12] M. Elamassie, M. Karbalayghareh, F. Miramirkhani, R. C. Kizilirmak, and M. Uysal, "Effect of fog and rain on the performance of vehicular visible light communications," in 2018 IEEE 87th Vehicular Technology Conference (VTC Spring), June 2018, pp. 1-6. 